\section{Mobile Brechanlage mit Strom aus dem Netz direkt an der Bruchwand, geliefert von SBM Mineral Processing GmbH an die Firma Geiger GmbH!}

Die SBM Mineral Processing $\mathrm{GmbH}$ bündelt seit Beginn des Jahres 2013 die Aktivitäten von MFL und SBM auf dem Gebiet der Aufbereitungstechnik.

Anfang 2013 wurden Vertrieb und Konstruktion der MFL-Sparte Aufbereitungstechnik in die SBM Mineral Processing $\mathrm{GmbH}$ eingegliedert. Die Fertigung der SBM-Produkte erfolgt in der Maschinenfabrik Liezen und Gießerei Ges.m.b.H. Mit diesem Schritt ist der Integrationsprozess der beiden Unternehmen nun abgeschlossen.

Im Jahre 2012 trat die Firma Geiger für den Quarzporphyrtuffsteinbruch im sächsischen Leukersdorf mit folgenden Vorgaben an die SBM MineralProcessing heran:

Gefordert war die Umstellung von stationärer auf mobile Aufbereitung zum Vorbrechen des gesprengten Hauwerkes auf 0-45 mm Endkörnung. Die Produktion sollte mindestens $250 \mathrm{t} / \mathrm{h}$ betragen und an der Bruchwand auf der Steinbruchsohle mit elektrischem Betrieb durch Netzstrom erfolgen. Die mobile Anlage sollte die bestehende stationäre, 1991 mit SBM-Ausrüstung errichtete Brechanlage ergänzen und zum Teil ersetzen.

Als Vorbrecher wurde die leistungsstarke Prallbrecheranlage $\mathrm{RCl}$ $130.130 / T-V$ von MFL vorgeschlagen. Auf Kundenwunsch wurde der Aufgabebunker auf $12 \mathrm{~m}^{3}$ Fassungsvolumen vergrößert, damit der Prallbrecher konstant mittels Radlader beschickt werden kann.
Um eine Durchsatzrate von $250 \mathrm{t} / \mathrm{h}$ der Körnung 0-45 mm sicherzustellen, muss der Brecher inklusive der Umlauflast eine Aufgabemenge von bis zu $360 \mathrm{t} / \mathrm{h}$ bewältigen können.

Die Anlage wurde durch ein radmobiles Sieb mit einer Maschenweite von $45 \mathrm{~mm}$ komplettiert, wobei ein Vierlager-Kurbelschwingsieb aufgrund der besonderen Eignung für diesen Einsatzfall gewählt wurde. Diese Maschine ist mit der Brechanlage verkettet, sowohl bezüglich der Energieversorgung als auch der Steuerung!

Die Anlage kann auch mit dem auf die Brechanlage aufgebauten Dieselaggregat betrieben werden, was im Probebetrieb der Anlage auch geschah. Damals waren die Betreiber vom geringen Dieselverbrauch der Anlage von nur $43 \mathrm{l} / \mathrm{h}$ sehr überrascht (Abb. 1).

Danach erfolgte die Einbindung in das Stromnetz, welche sehr einfach durch Netz-Generator-Umschaltung auf ein zweites Einspeiseklemmensystem verwirklicht wurde.

Während der Sprengung werden die Anlagen an einen sicheren Ort im Steinbruch gefahren und anschließend erneut in Hauwerksnähe platziert. Dieser An- und Abtransport dauert nicht mehr als 3 Stunden. Durch die Nähe der Bruchwand können die Radlader des Kunden optimal eingesetzt werden.

Die Betreiber sind mit der Betriebssicherheit, dem Verschleißverhalten, den einfachen Wartungsmöglichkeiten sowie mit der Leistung und Transportierbarkeit der Anlage sehr zufrieden.

Die Berücksichtigung der Sonderwünsche des Kunden durch den Lieferanten SBM führten zu einer maßgeschneiderten Lösung, die voll den Erwartungen der Fa. Geiger entspricht.
Dipl.-Ing. Werner Präsoll

SBM Mineral Processing GmbH

Oberweis 401

4664 Oberweis, Österreich

Büro Liezen

Werksstraße 5

8940 Liezen, Österreich

E-Mail:w.praesoll@mfl.at

Abb. 1: Mobile Brechanlage R-Cl 130 130/T-V mit nachgeschalteter Siebanlage im Steinbruch der Firma Geiger Leukersdorf/Sachsen, Deutschland

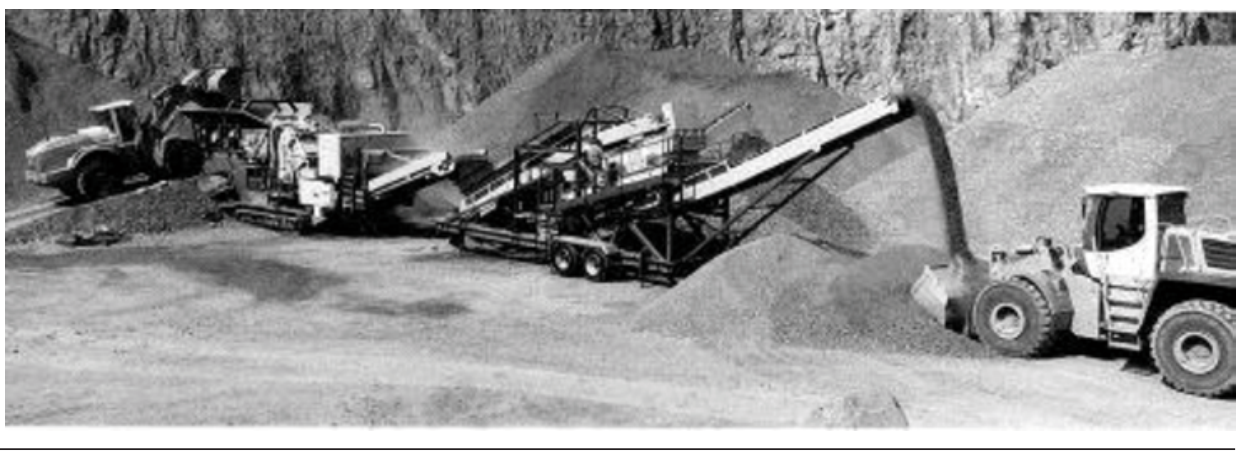

